\title{
Electrodynamics of Moving Anisotropic Media: The First-Order Theory ${ }^{1}$
}

\author{
C. T. Tai \\ Contribution From the University of Michigan Radiation Laboratory, Ann Arbor, Mich. \\ (Received October 6, 1964; revised November 9, 1964)

\begin{abstract}
Minkowski's theory of moving media is extended hereby to the anisotropic case. The corresponding Maxwell-Minkowski equations have been derived under the condition that the velocity of the moving medium is small compared to the velocity of light. As an application of that theory, it is shown that the characteristics of a plane wave propagating in a drifting magneto-ionic plasma can conveniently be determined from the constitutive parameters of the plasma without drifting. The equivalence between the convection current model adopted by Bell and Helliwell and the polarization current model suggested by Unz and correctly interpreted by Epstein, Bell, Smith, and Brice is also
\end{abstract} \\ pointed out.
}

\section{Introduction}

The foundation of the electrodynamics of moving media was laid by Minkowski [1908] and based upon the special theory of relativity. For a moving isotropic medium, the complete treatment is given by Sommerfield [1952]. When the velocity of the moving isotropic medium is small compared to the velocity of light, the resultant wave equations can be greatly simplified [Tai, 1964b]. Several technical problems arising from these wave equations have already been investigated [Compton and Tai, 1964; Collier and Tai, 1964; and Tai, 1964a].

In this report we shall present an extension of Minkowski's theory to moving anisotropic media. The theory is finally applied to a drifting magneto-ionic medium to determine the normal modes of plane waves which can exist in such a medium. The characteristic equation for the index of refraction so obtained by the first order relativistic transform method is the same as the one first derived by Bell and Helliwell [1959] using the convection current model and later by Epstein, Bell, Smith, and Brice [1963] using the polarization current model suggested by Unz [1962]. The equivalence between these models can be traced through Minkowski's theory.

\subsection{First-Order Relativistic Transformation of Field Vectors}

In this section, we shall first review the relativistic transformation of the field vectors defined in Maxwell's equations as first recognized by Einstein and later elaborated by Minkowski. Two inertial systems $(x, y, z)$ and $\left(x^{\prime}, y^{\prime}, z^{\prime}\right)$ are in relative motion. The primed system is assumed to be moving with a velocity $v$ with respect to the unprimed system. The time variable in the two systems will be denoted, respectively, by $t$ and $t^{\prime}$. Maxwell's equations and the equation of continuity defined in the systems are

$$
\begin{gathered}
\nabla^{\prime} \times \bar{E}^{\prime}=-\frac{\partial \bar{B}^{\prime}}{\partial t^{\prime}} \\
\nabla^{\prime} \times \bar{H}^{\prime}=\bar{J}^{\prime}+\frac{\partial \bar{D}^{\prime}}{\partial t^{\prime}} \\
\nabla^{\prime} \cdot \bar{J}^{\prime}=-\frac{\partial \rho^{\prime}}{\partial t^{\prime}} \\
\nabla \times \bar{E}=-\frac{\partial \bar{B}}{\partial t} \\
\nabla \times \bar{H}=J+\frac{\partial \bar{D}}{\partial t} \\
\nabla \cdot \bar{J}=-\frac{\partial \rho}{\partial t} .
\end{gathered}
$$

${ }^{1}$ The research reported in this paper was supported in part by the National Aeronautics and Space Administration under Grant NsG 444. 
If the velocity $v$ is small compared to the velocity of light, the first-order relativistic transformation between the two sets of field vectors, resulting from neglecting terms of the order of $(v / c)^{2}$, is given by [Cullwick, 1957]

$$
\begin{aligned}
& \bar{D}^{\prime}=\bar{D}+\frac{1}{c^{2}} \bar{v} \times \bar{H} \\
& \bar{E}^{\prime}=\bar{E}+\bar{v} \times \bar{B} \\
& \bar{B}^{\prime}=\bar{B}-\frac{1}{c^{2}} \bar{v} \times \bar{E} \\
& \bar{H}^{\prime}=\bar{H}-\bar{v} \times \bar{D} \\
& \bar{J}^{\prime}=\bar{J}-\rho \bar{v} \\
& \rho^{\prime}=\rho-\frac{1}{c^{2}} \bar{v} \cdot \bar{J}
\end{aligned}
$$

where

$$
c^{2}=1 / \mu_{0} \epsilon_{0}
$$

If two material field vectors $\bar{P}$ and $\bar{M}$ are used, such that one writes

$$
\begin{aligned}
& \bar{D}=\epsilon_{0} \bar{E}+\bar{P} \\
& \bar{B}=\mu_{0}(\bar{H}+\bar{M})
\end{aligned}
$$

and similarly for the primed quantities, then the firstorder relativistic transformation of the material field vectors can be deduced from (7-10). They are

$$
\begin{aligned}
& \bar{P}^{\prime}=\bar{P}-\frac{1}{c^{2}} \bar{v} \times \bar{M} \\
& \bar{M}^{\prime}=\bar{M}+\bar{v} \times \bar{P} .
\end{aligned}
$$

\subsection{First-Order Constitution Relations Between the Field Vectors Defined in the Unprimed System}

Let us assume that constitutive relations for an anisotropic medium in the primed system are known. In the most general case, they are

$$
\begin{aligned}
& \bar{D}^{\prime}=\overline{\overline{\epsilon^{\prime}}} \cdot \overline{E^{\prime}} \\
& \bar{B}^{\prime}=\overline{\bar{\mu}} \cdot \bar{H}^{\prime} \\
& \bar{J}^{\prime}=\overline{\overline{\sigma^{\prime}}} \cdot \bar{E}^{\prime},
\end{aligned}
$$

where the sign $"="$ is used to denote a dyadic. If the medium is dispersive, it is understood that $\overline{\bar{\epsilon}}^{\prime}, \overline{\bar{\mu}}^{\prime}$, and $\overline{\bar{o}}^{\prime}$ would be functions of the frequency defined in the primed system, which will be denoted by $\omega^{\prime}$. By substituting (7) to (11) into (18) to (20), we obtain

$$
\begin{aligned}
& \bar{D}+\frac{1}{c^{2}} \bar{v} \times \bar{H}=\overline{\overline{\epsilon^{\prime}}} \cdot(\bar{E}+\bar{v} \times \bar{B}) \\
& \bar{B}-\frac{1}{c^{2}} \bar{v} \times \bar{E}=\overline{\overline{\mu^{\prime}}} \cdot(\bar{H}-\bar{v} \times \bar{D}) \\
& \bar{J}-\rho \bar{v}=\overline{\overline{\sigma^{\prime}}} \cdot(\bar{E}+\bar{v} \times \bar{B})
\end{aligned}
$$

By eliminating $\bar{D}$ or $\bar{B}$ from (2l) and (22) and neglecting terms of the order of $(v / c)^{2}$, we have

$$
\begin{aligned}
& \bar{D}=\overline{\overline{\epsilon^{\prime}}} \cdot\left[\bar{E}+\bar{v} \times\left(\overline{\overline{\mu^{\prime}}} \cdot \bar{H}\right)\right]-\frac{1}{c^{2}} \bar{v} \times \bar{H} \\
& \bar{B}=\overline{\overline{\mu^{\prime}}} \cdot\left[\bar{H}-\bar{v} \times\left(\overline{\overline{\epsilon^{\prime}}} \cdot \bar{E}\right)\right]+\frac{1}{c^{2}} \bar{v} \times \bar{E} \\
& \bar{J}=\overline{\overline{\sigma^{\prime}}} \cdot\left[\bar{E}+\bar{v} \times\left(\overline{\overline{\mu^{\prime}}} \cdot \bar{H}\right)\right]+\rho \bar{v} .
\end{aligned}
$$

Since $\nabla \cdot \bar{D}=\rho,(26)$ may be written in the form

$$
\bar{J}=\overline{\overline{\sigma^{\prime}}} \cdot\left[\bar{E}+\bar{v} \times\left(\overline{\overline{\mu^{\prime}}} \cdot \bar{H}\right)\right]+\bar{v} \nabla \cdot\left(\overline{\overline{\epsilon^{\prime}}} \cdot \bar{E}\right) .
$$

Again, terms of the order of $(v / c)^{2}$ have been neglected in deriving (27). Equations (24), (25), and (27) contain the first-order constitutive relations between the field quantities defined in the unprimed system in terms of the constitutive parameters known or given in the primed system. By substituting these equations into (4) and (5), we obtain the Maxwell-Minkowski wave equations for the field vectors $\bar{E}$ and $\bar{H}$ in a moving anisotropic medium which represent a formal extension of Minkowski's theory of moving media to the anistropic case.

\section{Magneto-Ionic Theory for Drifting Plasma}

In this section, we shall apply the above formulation to derive the characteristic equation for the index of refraction for a plane wave propagating in a drifting magneto-ionic medium. The convection current model will be treated first. In such a model the plasma is assumed to consist of free electrons with a drift velocity $\bar{v}$. The primed system is attached to the drifting electrons and the unprimed system is fixed to an observer. According to the well-known theory of a stationary magneto-ionic media, the relation between the convection current $\overline{J^{\prime}}$ and a harmonically oscillating. electric field $\bar{E}^{\prime}$ with frequency $\omega^{\prime}$ can be written in the form

$$
\overline{\overline{r^{\prime}}} \cdot \overline{J^{\prime}}=\bar{E}^{\prime}
$$

where the dyadic $\overline{\overline{r^{\prime}}}$ is given by

$$
\overline{\overline{r^{\prime}}}=\frac{j}{X^{\prime} \omega^{\prime} \epsilon_{0}}\left[\left(1-j Z^{\prime}\right) \overline{\bar{I}}+j \overline{\bar{Y}} \bar{Y}^{\prime}\right]
$$


The parameters contained in (29) are defined as follows:

$$
\begin{aligned}
& X^{\prime}=\frac{N|e|^{2}}{m \omega^{\prime 2} \epsilon_{0}}=\left(\frac{\omega_{p}}{\omega^{\prime}}\right)^{2}, \\
& \omega_{p}=\text { plasma frequency } \\
& Z^{\prime}=\frac{\omega_{c}}{\omega^{\prime}}, \\
& \omega_{c}^{\prime}=\text { collision frequency, } \\
& \overline{\bar{Y}}^{\prime}=\left[\begin{array}{rcc}
0 & -Y_{z}^{\prime} & Y_{y}^{\prime} \\
Y_{z}^{\prime} & 0 & -Y_{x}^{\prime} \\
-Y_{y}^{\prime} & Y_{x}^{\prime} & 0
\end{array}\right] \\
& \bar{Y}^{\prime}=Y_{x}^{\prime} \hat{x}+Y_{y}^{\prime} \hat{y}+Y_{z}^{\prime} \hat{z}=\frac{\mu_{0}|e| \bar{H}_{0}}{m \omega^{\prime}} \\
& =\frac{\bar{\omega}_{m}}{\omega^{\prime}}, \\
& \omega_{m}=\text { gyromagnetic frequency. }
\end{aligned}
$$

The dyadic $\overline{\overline{r^{\prime}}}$ is clearly the recriprocal of $\overline{\overline{\sigma^{\prime}}}$ previously introduced in (20), i.e.,

$$
\overline{\overline{\sigma^{\prime}}} \cdot \overline{\overline{r^{\prime}}}=\overline{\bar{I}}
$$

In the convection current model, the medium is considered to be unpolarized and unmagnetized, hence,

$$
\begin{aligned}
& \overline{\overline{\epsilon^{\prime}}}=\epsilon_{0} \overline{\bar{I}} \\
& \overline{\overline{\mu^{\prime}}}=\mu_{0} \overline{\bar{I}} .
\end{aligned}
$$

Equations (24), (25), and (27), therefore, reduce to

$$
\begin{gathered}
\bar{D}=\epsilon_{0} \bar{E} \\
\bar{B}=\mu_{0} \bar{H} \\
\overline{r^{\prime}} \cdot\left[\bar{J}-\bar{v} \nabla \cdot\left(\mu_{0} \bar{E}\right)\right]=\bar{E}+\mu_{0} \bar{v} \times \bar{H} .
\end{gathered}
$$

On account of (33) and (34), (4) and (5) become, for a harmonically oscillating field,

$$
\begin{aligned}
& \nabla \times \bar{E}=-j \omega \mu_{0} \bar{H} \\
& \nabla \times \bar{H}=\bar{J}+j \omega \epsilon_{0} \bar{E} .
\end{aligned}
$$

Equations (35) through (37) are the three basic equations relating $\bar{E}, \bar{H}$, and $\bar{J}$ in a drifting magneto-ionic medium. They have been derived by applying Minkowski's first-order relativistic transform method.

To derive the characteristic equation for the index of refraction, let us assume a harmonically oscillating plane wave to be propagating in the $z$-direction in the unprimed system so that all the field components have a dependence of $e^{j(\omega t-k z)}$. The wave number $k$, the index of refraction $n$, the phase velocity $V_{p}$ and the angular frequency $\omega$ are related as follows:

$$
k=\frac{\omega}{V_{p}}=\frac{n \omega}{c} .
$$

As a result of the assumption of a plane wave propagating in the $z$-direction, (36) and (37) can be written as

$$
\begin{aligned}
& -j k \hat{z} \times \bar{E}=-j \omega \mu_{0} \bar{H} \\
& -j k \hat{z} \times \bar{H}=\bar{J}+j \omega \epsilon_{0} E .
\end{aligned}
$$

Eliminating $\bar{H}$ between (39) and (40), we obtain

$$
\bar{J}=-j \omega \epsilon_{0}\left[\left(1-n^{2}\right) \bar{E}+n^{2} E_{z} \hat{z}\right] .
$$

Substituting the expressions for $\bar{H}$ and $\bar{J}$ as given by (39) and (41) into (35), we obtain a homogeneous equation for $\bar{E}$

$$
-j \omega_{0} \overline{\overline{r^{\prime}}} \cdot\left[\left(1-n^{2}\right) \bar{E}+\left(n^{2} \hat{z}-n \bar{\beta}\right) E_{z}\right]=\bar{E}+n \bar{\beta} \times(\hat{z} \times \bar{E})
$$

where

$$
\bar{\beta}=\bar{v} / c
$$

To find the characteristic equation for $n$, the primed quantities contained in $\overline{\bar{r}^{\prime}}$ must first be converted into explicit functions of $n$. Because of the Doppler shift, the relationship between $\omega^{\prime}$ and $\omega$, accurate to the order of $v / c$, is given by

$$
\begin{aligned}
\omega^{\prime} & =\omega\left(1-\frac{k}{\omega} v_{z}\right) \\
& =\omega\left(1-\frac{n}{c} v_{z}\right) \\
& =\omega\left(1-n \beta_{z}\right) .
\end{aligned}
$$

The parameters contained in $r^{\prime}$, therefore, can be written as:

$$
\begin{gathered}
X^{\prime}=\left(\frac{\omega_{p}}{\omega^{\prime}}\right)^{2}=\frac{1}{\left(1-n \beta_{z}\right)^{2}}\left(\frac{\omega_{p}}{\omega}\right)^{2}=\frac{X}{\left(1-n \beta_{z}\right)^{2}} \\
Z^{\prime}=\frac{\omega_{c}}{\omega^{\prime}}=\frac{1}{1-n \beta_{z}}\left(\frac{\omega_{c}}{\omega}\right)=\frac{Z}{1-n \beta_{z}}, \\
\bar{Y}^{\prime}=\frac{\bar{\omega}_{m}}{\omega^{\prime}}=\frac{1}{1-n \beta_{z}}\left(\frac{\bar{\omega}_{m}}{\omega}\right)=\frac{\bar{Y}}{1-n \beta_{z}} .
\end{gathered}
$$


hence,

$$
\begin{aligned}
-j \omega \epsilon_{0} \overline{\bar{r}}^{\prime} & =\frac{1-n \beta_{z}}{X}\left[\left(1-j \frac{Z}{1-n \beta_{z}}\right) \overline{\bar{I}}+j \frac{\overline{\bar{Y}}}{1-n \beta_{z}}\right] \\
& =\frac{1}{X}\left(1-n \beta_{z}-j Z\right) \overline{\bar{I}}+j \overline{\bar{Y}} .
\end{aligned}
$$

On account of (48), the explicit expression for (42) is given by

$$
\begin{gathered}
{\left[\left(1-n \beta_{z}-j Z\right) \overline{\bar{I}}+j \overline{\bar{Y}}\right] \cdot\left[\left(1-n^{2}\right) \bar{E}+\left(n^{2} \hat{z}-n \bar{\beta}\right) E_{Z}\right]} \\
=X[\bar{E}+n \bar{\beta} \times(\hat{z} \times \bar{E})] .
\end{gathered}
$$

Equation (49) can be written in the form

$$
\overline{\bar{A}} \cdot \bar{E}=0 \text {. }
$$

Where the element of the dyadic $\overline{\bar{A}}$ are found to be

$$
\begin{aligned}
& A_{x x}=\left(n^{2}-1\right)\left(1-n \beta_{z}-j Z\right)+\left(1-n \beta_{z}\right) X \\
& A_{x y}=-j\left(n^{2}-1\right) Y_{z} \\
& A_{x z}=\left(1-n \beta_{z}-j Z\right) n \beta_{x}-j n \beta_{y} Y_{z}-j\left(1-n \beta_{z}\right) Y_{y} \\
& A_{y x}=j\left(n^{2}-1\right) Y_{z} \\
& A_{y y}=\left(n^{2}-1\right)\left(1-n \beta_{z}-j Z\right)+\left(1-n \beta_{z}\right) X \\
& A_{y z}=\left(1-n \beta_{z}-j Z\right) n \beta_{z}+j n \beta_{x} Y_{z}+j\left(1-n \beta_{z}\right) Y_{x} \\
& A_{z x}=-j\left(n^{2}-1\right) Y_{y}+n \beta_{x} X \\
& A_{z y}=j\left(n^{2}-1\right) Y_{x}+n \beta_{y} X \\
& A_{z z}=-\left(1-n \beta_{z}\right)\left(1-n \beta_{z}-j Z\right)-j n \beta_{x} Y_{y}+j n \beta_{y} Y_{x}+X .
\end{aligned}
$$

By setting the determinant of $A$ equal to zero, one obtains the characteristic equation for $n$. Equation (50) is the same as the one first obtained by Bell and Helliwell $[1959]^{2}$ if we discard in their work the part contributed by the ambient plasma. It is also identical to the one obtained by Epstein, Bell, Smith, and Brice [1963] after correctly interpreting the work of Unz [1962]. The alternative derivation presented here appears to provide a clearer view as to how the original Appleton-Hartree matrix was transformed as a result of the motion. It also shows another application of Minkowski's powerful theory. While the treatment of Bell and Helliwell may be considered as "classical" if one accepts the Lorentz force equation as a prere-

${ }^{2}$ Bell and Helliwell considered only the case where $\bar{v}$ and $\bar{H}_{0}$ have the same direction. This restriction can easily be removed. They also included a stationary plasma in addition to the drifting one. The part contributed by the stationary plasma can be included in our treatment if necessary. There are some minor algebraic mistakes in Bell and Helliwell's work. For example the matrix for $\bar{J}_{M 1}$, equation (12), contains a parameter $p_{1}$ which is defined as $k / k_{1} Y_{L}$ that appears to be incorrect. The parameter $l^{2}$ defined in the list of symbols does not seem to fit the dimensional analysis. lativistic postulate, as we normally do, instead of as a consequence of the relativistic transformation, the treatment of Unz, Epstein, et al., is not. This is because the $E$ and $H$ fields in the EPHMv formulation are only partial fields [1964b]. The force equation in that formulation therefore does not have the same structure as the Lorentz force equation. In order to justify that treatment, we shall treat the same problem based upon the polarization current model and point out its implications.

\section{Polarization Current Model}

In the book by Ratcliffe [1959], the AppletonHartree equation for a stationary magneto-ionic medium was derived by considering the medium to be made of polarized matter or bound electrons. We shall call such a model "the polarization current model" in contrast to the convection current model presented in the previous section. In the polarization current model, one has

$$
\begin{aligned}
\bar{J}^{\prime} & =0 \\
\bar{M}^{\prime} & =0
\end{aligned}
$$

and

$$
\overline{P^{\prime}}=\epsilon_{0} \overline{\overline{\chi^{\prime}}} \cdot \overline{E^{\prime}}
$$

where, numerically, the dyadic susceptibility $\overline{\overline{\chi^{\prime}}}$ is related to $\overline{\bar{\sigma}^{\prime}}$ defined by (30) in the following way

$$
j \omega^{\prime} \epsilon_{0} \overline{\overline{\chi^{\prime}}}=\overline{\overline{\sigma^{\prime}}} .
$$

As a result of using $\bar{P}^{\prime},(24)$ and (25) become

$$
\begin{aligned}
& \bar{D}=\overline{\overline{\epsilon^{\prime}}} \cdot\left(\bar{E}+\mu_{0} \bar{v} \times \bar{H}\right)-\frac{1}{c^{2}} \bar{v} \times \bar{H} \\
& \bar{B}=\mu_{0}\left[\bar{H}-\bar{v} \times\left(\overline{\overline{\epsilon^{\prime}}} \cdot \bar{E}\right)\right]+\frac{1}{c^{2}} \bar{v} \times \bar{E}
\end{aligned}
$$

where

$$
\overline{\overline{\epsilon^{\prime}}}=\epsilon_{0}\left(\overline{\bar{I}}+\overline{\overline{\chi^{\prime}}}\right) .
$$

Two alternative expressions for (56) and (57) are

$$
\begin{aligned}
& \bar{D}=\epsilon_{0} \bar{E}+\epsilon_{0} \overline{\overline{\chi^{\prime}}} \cdot\left(\bar{E}+\mu_{0} \bar{v} \times \bar{H}\right) \\
& \bar{B}=\mu_{0} \bar{H}-\mu_{0} \bar{v} \times\left(\epsilon_{0} \overline{\overline{\chi^{\prime}}} \cdot \bar{E}\right) .
\end{aligned}
$$

If we denote

$$
\left.\epsilon_{0} \overline{\overline{\chi^{\prime}}} \cdot \overline{(E}+\mu_{0} \bar{v} \times \bar{H}\right)=\bar{P}
$$


then

$$
\begin{aligned}
& \bar{D}=\epsilon_{0} \bar{E}+\bar{P} \\
& \bar{B}=\mu_{0}(\bar{H}-\bar{v} \times \bar{P}) .
\end{aligned}
$$

Within the order of accuracy of $v / c$, the apparent difference between (60) and (63) is of no consequence. The basic equations involved in the polarization current model are summarized below:

$$
\begin{aligned}
& \nabla \times \bar{E}=-j \omega \mu_{0}(\bar{H}-\bar{v} \times \bar{P}) \\
& \nabla \times \bar{H}=j \omega\left(\epsilon_{0} \bar{E}+\bar{P}\right) \\
& \bar{P}=\epsilon_{0} \overline{\overline{\chi^{\prime}}} \cdot\left(\bar{E}+\mu_{0} \bar{v} \times \bar{H}\right) .
\end{aligned}
$$

These equations play a role similar to (35) through (37) in the convection current model. As far as the result is concerned it will be shown that two models do yield the same answer as far as the characteristic equation for $n$ is concerned. Equations (64) through (66) may be transformed into another form by introducing a field vector $\bar{H}_{p}$ defined by

$$
\bar{H}_{p}=\bar{H}-\bar{v} \times \bar{P}
$$

that gives

$$
\begin{gathered}
\nabla \times \bar{E}=-j \omega \mu_{0} \bar{H}_{p} \\
\nabla \times \bar{H}_{p}=j \omega\left(\epsilon_{0} \bar{E}+\bar{P}\right)-\nabla \times(\bar{v} \times \bar{P}) \\
\bar{P}=\epsilon_{0} \overline{\overline{\chi^{\prime}}} \cdot\left(\bar{E}+\mu_{0} \bar{v} \times \bar{H}_{p}\right) .
\end{gathered}
$$

A term of the order of $(v / c)^{2}$ is discarded in converting (66) into (70). Equations (68) and (69) are identical in form to the two equations used by Unz [1962] who originally investigated that problem based upon the so-called EHPMv formulation. Equation (70) can be transformed to the force equation interpreted by Epstein [1963]. Thus, if we write (70) in the form

$$
j \omega^{\prime} \bar{P}=\overline{\overline{\sigma^{\prime}}} \cdot\left(\bar{E}+\mu_{0} \bar{v} \times \bar{H}_{p}\right)
$$

or

$$
j \omega^{\prime} \overline{\overline{r^{\prime}}} \cdot \bar{P}=\left(\bar{E}+\mu_{0} \bar{v} \times \bar{H}_{p}\right) .
$$

Using $\overline{\overline{r^{\prime}}}$ given by (29) and substituting it into (72) we may reconstruct the differential equation corresponding to (72). We consider

$$
\frac{-1}{\epsilon_{0} X^{\prime}}\left[\left(1-j Z^{\prime}\right) \overline{\bar{I}}+j \overline{\bar{Y}} \bar{Y}^{\prime}\right] \cdot \bar{P}=\bar{E}+\mu_{0} \bar{v} \times \bar{H}_{p}
$$

which is the same as

$$
\frac{-m \omega^{\prime 2}}{N|e|^{2}}\left[\left(1-j \frac{\omega_{c}^{\prime}}{\omega^{\prime}}\right) \bar{P}+j \frac{\mu_{0}|e| \bar{H}_{0}}{m \omega^{\prime}} \times \bar{P}\right]=\bar{E}+\mu_{0} \bar{v} \times \bar{H}_{p}
$$

If we let

$$
\bar{P}=-N e \bar{R}_{p}
$$

then, (74) is equivalent to

$$
m \frac{\partial^{2} \bar{R}_{p}}{\partial t^{\prime 2}}+m \omega_{c}^{\prime} \frac{\partial \bar{R}_{p}}{\partial t^{\prime}}=-e\left(\bar{E}+\mu_{0} \frac{\partial \bar{R}_{p}}{\partial t^{\prime}} \times \bar{H}_{0}+\mu_{0} \bar{v} \times \bar{H}_{p}\right) .
$$

Since $t^{\prime}$ is defined in the primed system, the partial derivation of a function with respect to $t^{\prime}$ is the same as the material derivative $\frac{d}{d t}=\frac{\partial}{\partial t}+(\bar{v} \cdot \nabla)$ evaluated in the unprimed system as a result of the first-order Lorentz transformation, i.e., neglecting terms of the order of $(v / c)^{2}$. Equation (76), therefore, is identical to the force equation in the EHPMv formulation. As we have mentioned earlier, as far as the characteristic equation for $n$ is concerned, both the convectioncurrent model and the polarization model provide the same answer. However, it is obvious from this discussion that there are several delicate concepts involved in the polarization current model. It appears that these concepts and their acceptance can best be understood and justified with the aid of Minkowski's theory. Finally, it should be pointed out that although the classical method provides the correct answer when $(v / c)^{2}<<1$ it fails when $v$ is comparable to $c$. In that case, one must depend upon Minkowski's exact theory to formulate such a problem.

\section{References}

Bell, T. F., and R. A. Helliwell (Sept. 1959), Traveling-wave amplification in the ionosphere, Stanford University, Radio Propagation Laboratory Techn. Report No. 2.

Bell, T. F., R. Smith, and N. Brice (Mar. 1963), Comments on the magnetoionic theory for a drifting plasma, IEEE Trans. Ant. Prop. AP-11, 194-195.

Collier, J. R., and C. T. Tai (May 1964), Propagation of plane waves in lossy moving media, IEEE Trans. Ant. Prop. AP-12, 375.

Compton, R. T. Jr., and C. T. Tai (Mar. 1964), Poynting's theorem for radiating systems in moving media, IEEE Trans. Ant. Prop. AP-12, 238.

Cullwick, E. G. (1957), Electromagnetism and relatively, 2d ed. (Longmans, Green and Company Ltd., London).

Epstein, Melvin (Mar. 1963), Comments on two papers dealing with EM propagation in moving plasma, IEEE Trans. Ant. Prop. AP-1 1, 193-194.

Minkowski, Herman (1908), Die Grundgleichungen fur die elektromagnetischen Vorgange in bewegten Kerpern, pp. 53-116, Gottingen Nachrichen.

Ratcliffe, J. A. (1959), The magnetoionic theory and its applications to the ionosphere (Cambridge University Press, Cambridge, Mass.).

Sommerfeld, A. (1952), Electrodynamics (Academic Press Inc., New York, N.Y.).

Tai, C. T. (May 1964a), Two scattering problems involving moving media, Techn. Report 1991-7, Antenna Laboratory, Ohio State University.

Tai, C. T. (June 1964b), A study of electrodynamics of moving media, Proc. IEEE 52, 685-689.

Unz, H. (July 1962), The magnetoionic theory for drifting plasma, IEEE Trans. Ant. Prop. AP-1 0, 459-464.

(Paper 69D3-477) 